\title{
The CUORE Detector and Results
}

Irene Nutini ${ }^{1,2}$ D D. Q. Adams ${ }^{3} \cdot$ C. Alduino ${ }^{3} \cdot$ K. Alfonso ${ }^{4} \cdot$ F. T. Avignone III $^{3}$.

O. Azzolini ${ }^{5}$ - G. Bari ${ }^{6}$ - F. Bellini ${ }^{7,8}$ - G. Benato ${ }^{9} \cdot$ M. Biassoni ${ }^{2} \cdot$ A. Branca ${ }^{1,2}$.

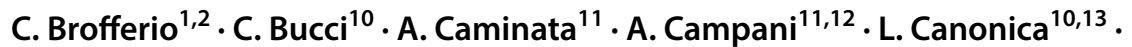

X. G. Cao ${ }^{14}$ - S. Capelli, $i^{1,2}$ L. Cappelli, $i^{9,10,15}$. L. Cardani ${ }^{8}$ - P. Carniti ${ }^{1,2} \cdot$ N. Casali ${ }^{8}$.

D. Chiesa ${ }^{1,2} \cdot$ N. Chott $^{3} \cdot$ M. Clemenza ${ }^{1,2} \cdot$ S. Copello ${ }^{10,16} \cdot$ C. Cosmelli $^{7,8}$.

O. Cremonesi ${ }^{2}$ - R. J. Creswick ${ }^{3}$ - A. D'Addabbo ${ }^{10}$ - D. D'Aguanno ${ }^{10,17}$ - I. Dafinei ${ }^{8}$.

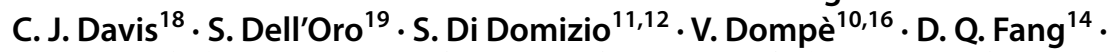

G. Fantini ${ }^{10,16} \cdot$ M. Faverzani ${ }^{1,2} \cdot$ E. Ferri ${ }^{1,2} \cdot$ F. Ferroni $^{8,16} \cdot$ E. Fiorini ${ }^{1,2}$.

M. A. Franceschi ${ }^{20}$ - S. J. Freedman ${ }^{9,15}$ - B. K. Fujikawa ${ }^{15}$ - A. Giachero ${ }^{1,2}$.

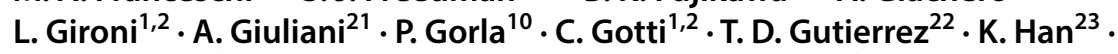

K. M. Heeger ${ }^{18} \cdot$ R. G. Huang ${ }^{9} \cdot$ H. Z. Huang ${ }^{4} \cdot$ J. Johnston $^{13} \cdot$ G. Keppel ${ }^{5}$.

Yu. G. Kolomensky ${ }^{9,15}$. C. Ligi ${ }^{20}$. Y. G. Ma ${ }^{14}$ - L. Ma ${ }^{4}$. L. Marini ${ }^{9,15}$.

R. H. Maruyama ${ }^{18} \cdot$ Y. Mei $^{15} \cdot$ N. Moggi ${ }^{6,24}$ - S. Morganti ${ }^{8} \cdot$ T. Napolitano $^{20}$.

M. Nastasi ${ }^{1,2} \cdot$ J. Nikkel ${ }^{18}$. C. Nones ${ }^{25}$ - E. B. Norman ${ }^{26,27}$ - V. Novati ${ }^{21}$.

A. Nucciotti ${ }^{1,2} \cdot$ T. O'Donnell ${ }^{19} \cdot$ J. L. Ouellet ${ }^{13} \cdot$ C. E. Pagliarone ${ }^{10,17}$.

L. Pagnanini ${ }^{1,2} \cdot$ M. Pallavicini ${ }^{11,12} \cdot$ L. Pattavina ${ }^{10} \cdot$ M. Pavan ${ }^{1,2} \cdot$ G. Pessina ${ }^{2}$.

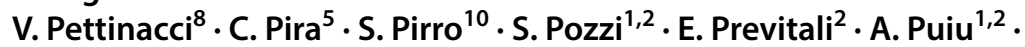

C. Rosenfeld ${ }^{3} \cdot$ C. Rusconi $i^{3,10} \cdot$ M. Sakai ${ }^{9} \cdot$ S. Sangiorgio ${ }^{26} \cdot$ B. Schmidt ${ }^{15}$.

N. D. Scielzo ${ }^{26} \cdot$ V. Singh ${ }^{9} \cdot$ M. Sisti ${ }^{1,2} \cdot$ D. Speller ${ }^{18} \cdot$ L. Taffarello $^{28}$.

F. Terranova ${ }^{1,2}$. C. Tomei $^{8} \cdot$ M. Vignati ${ }^{8}$ - S. L. Wagaarachchi $i^{9,15}$ - B. S. Wang ${ }^{26,27}$.

B. Welliver ${ }^{15} \cdot$ J. Wilson $^{3} \cdot \mathrm{K}$. Wilson ${ }^{3} \cdot$ L. A. Winslow ${ }^{13} \cdot$ L. Zanotti $^{1,2}$.

S. Zimmermann ${ }^{29} \cdot$ S. Zucchelli ${ }^{6,24}$

Received: 19 August 2019 / Accepted: 8 February 2020 / Published online: 24 February 2020

(c) Springer Science+Business Media, LLC, part of Springer Nature 2020

\section{Abstract}

The cryogenic underground observatory for rare events (CUORE) is a cryogenic experiment searching for neutrinoless double beta decay $(0 v \beta \beta)$ of ${ }^{130} \mathrm{Te}$. The detector consists of an array of $988 \mathrm{TeO}_{2}$ crystals arranged in a compact cylindrical structure of 19 towers. We report the CUORE initial operations and optimization campaigns. We then present the CUORE results on $0 v \beta \beta$ and $2 \nu \beta \beta$ decay of ${ }^{130} \mathrm{Te}$ obtained from the analysis of the physics data acquired in 2017.

Keywords Neutrinos $\cdot 0 \nu \beta \beta \cdot$ Macro-calorimeters $\cdot$ Cryogenics $\cdot$ CUORE

S. Freedman-deceased

Irene Nutini

irene.nutini@mib.infn.it

Extended author information available on the last page of the article 


\section{Double Beta Decay}

Double beta decay is a second-order weak decay where a nucleus $(\mathrm{A}, \mathrm{Z})$ undergoes two beta decays to its isobar $(\mathrm{A}, \mathrm{Z}+2)$ in a single step, emitting two electrons in the process. According to the standard model (SM), the process observes lepton number conservation and two electron anti-neutrinos are simultaneously emitted $(2 v \beta \beta)$. The SM extensions that accommodate for a Majorana neutrino nature predict a lepton number violating neutrinoless double beta decay channel in which only the two electrons are emitted $(0 v \beta \beta)$. The experimental signature of $0 v \beta \beta$ decay is a peak in the summed energy spectrum of the final state electrons at the $Q$-value of the decay $\left(Q_{\beta \beta}\right)$.

For the $2 v \beta \beta$ decay, several measurements of the half-lives have been made for few isotopes, giving values of the order of $T_{2 v \beta \beta}^{1 / 2} \sim 10^{18}-10^{24}$ year [1]. At the same time, there are several experiments hunting for $0 v \beta \beta$ process, but no evidence for it has been uncovered yet; thus, only lower limits on half-life of $0 \nu \beta \beta$ are provided, $T_{0 \vee \beta \beta}^{1 / 2}>10^{24}-10^{26}$ year [2]. In case of a light Majorana neutrino exchange mechanism for the $\beta \beta$ decay, the half-life of the $0 v \beta \beta$ process can be expressed as a function of the neutrino mass,

$$
\left[T_{0 \nu \beta \beta}^{1 / 2}\right]^{-1} \propto G_{0 \nu}\left|M_{0 \nu \beta \beta}\right|^{2}\left|m_{\beta \beta}\right|^{2}
$$

where $m_{\beta \beta}$ is usually referred to as the "effective Majorana mass," $G_{0 v}$ is the phase space factor and $\left|M_{0 \nu \beta \beta}\right|^{2}$ is the nuclear matrix element [3].

\section{The CUORE Experiment}

The Cryogenic Underground Observatory for Rare Events (CUORE) is a ton-scale cryogenic experiment. The main goal of the experiment is the investigation of the $0 v \beta \beta$ decay of ${ }^{130} \mathrm{Te}[4,5]$. The CUORE detector, shown in Fig. 1(left), is composed by $988 \mathrm{TeO}_{2}$ crystals arranged in a structure of 19 towers. The total mass of $\mathrm{TeO}_{2}$ is $742 \mathrm{~kg}$, corresponding to $206 \mathrm{~kg}$ of ${ }^{130} \mathrm{Te}$.

\section{1 $\mathrm{TeO}_{2}$ Macro-calorimeters}

The CUORE choice of using the ${ }^{130} \mathrm{Te}$ isotope to search for the $0 v \beta \beta$ process has been driven by several factors. This isotope has large transition energy, $Q_{\beta \beta}=2527.518 \mathrm{keV}$, and this helps in order to have a reduced background from natural radioactivity in the region of interest (ROI). It has also the highest natural isotopic abundance, $\eta=34.167 \%$, among the $\beta \beta$ emitters. This avoids the need for a costly and time-consuming isotopic enrichment.

The CUORE experiment employs a calorimetric phonon energy detection method and operates at temperatures of the order of $\sim 10 \mathrm{mK}$. This method is characterized by an extremely good energy resolution, which allows to consider a narrower ROI, and fewer background events will contend with the $0 v \beta \beta$ signal. However, it has to 

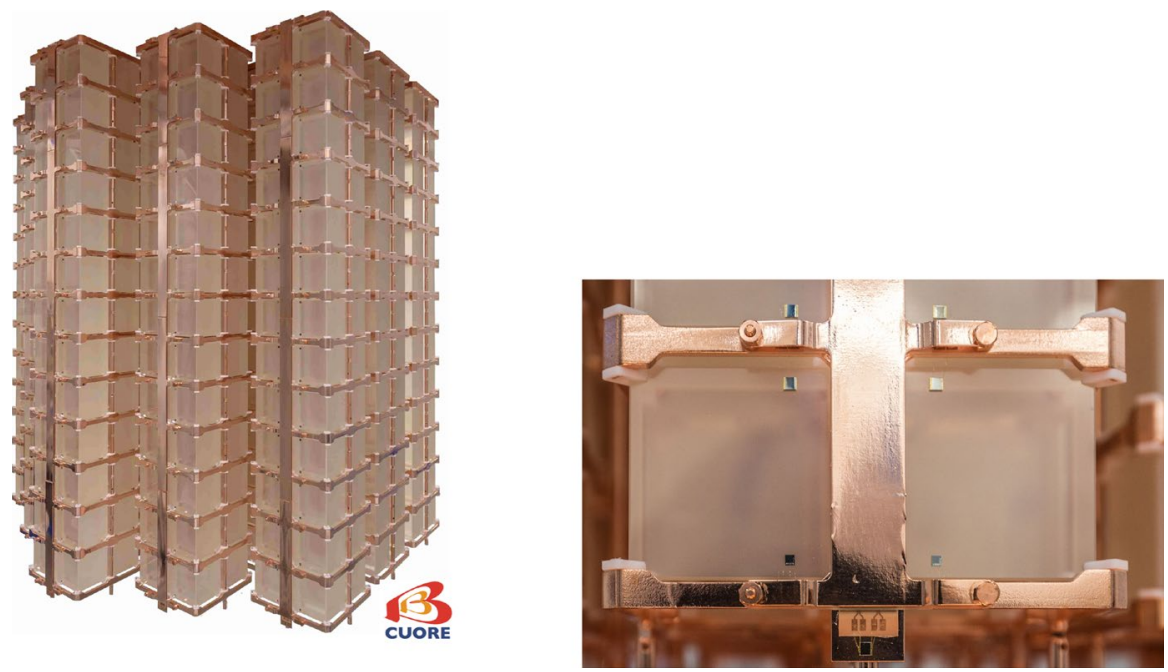

Fig. 1 (Left) Complete CUORE detector: 19 towers hosting the $988 \mathrm{TeO}_{2}$ crystals. (Right) CUORE instrumented detectors: $\mathrm{TeO}_{2}$ crystals held in the copper structure, Ge-NTD and Si heater chips are glued on the crystals (Color figure online)

be taken into account the technical difficulty of cooling a large mass detector to such a low temperature.

Cryogenic calorimeters made of materials containing $\beta \beta$-emitters were developed by the group of Fiorini [6] that mainly focused on the search for the $0 v \beta \beta$ of ${ }^{130} \mathrm{Te}$ using tellurium dioxide $\left(\mathrm{TeO}_{2}\right)$ crystals, which allows to have the ${ }^{130} \mathrm{Te}$ source within the detector absorber itself.

The CUORE $\mathrm{TeO}_{2}$ crystals [7] are $5 \times 5 \times 5 \mathrm{~cm}^{3}$ each and weigh an average of $750 \mathrm{~g}$ (see Fig. 1). They are made with ${ }^{\text {nat }} \mathrm{Te}$; each crystal contains $208 \mathrm{~g}$ of ${ }^{130} \mathrm{Te}$. Each $\mathrm{TeO}_{2}$ crystal is instrumented with a neutron transmutation-doped germanium (NTD-Ge) thermistor, which acts as a voltage transducer; the thermal phonons, produced by the energy release in the $\mathrm{TeO}_{2}$ crystals, are converted into a voltage pulse at the thermistor ends. Each crystal absorber is also instrumented with a silicon Joule heater, which is designed to periodically provide a fixed amount of energy in the detector for gain stabilization purposes. Copper strips connected to the NTD and heaters by gold wires are utilized for the signal readout. A dedicated low-noise circuit with two large load resistors maintained at room temperature provides the bias voltage to each NTD. High-precision calibration pulses are sent periodically to the heaters from pulser boards to study possible thermal instabilities of the system [8].

\subsection{Experimental Setup}

The CUORE experiment is hosted at the Laboratori Nazionali del Gran Sasso of the Istituto Nazionale di Fisica Nucleare (INFN) in central Italy. The Gran Sasso mountain provides the detector with an overburden of $1400 \mathrm{~m}$ of rock. The cosmic muon 
rate is reduced by 6 orders of magnitude relative to the surface; this creates appropriate conditions for rare event physics experiments, such as the search for $0 v \beta \beta$.

The CUORE $\mathrm{TeO}_{2}$ crystals have to be operated at a temperature of $\sim 10 \mathrm{mK}$ in order to behave as efficient calorimeters. A custom cryostat has been designed to house the ton-scale CUORE detector [9, 10]. Moreover, to avoid radioactive background, only a few construction materials were acceptable and lead shields were integrated in the structure. The cryogenic system implemented in CUORE has to cool down the $\mathrm{TeO}_{2}$ detectors, the cryostat components and the $\mathrm{Pb}$ shields and to maintain the detector volume stably at a temperature around $10 \mathrm{mK}$ for at least 5 years. A cryogen free refrigerator system is utilized for the cooling of the detector; it consists of three subsystem: the fast cooling system (FCS), five pulse tube cryocoolers (PTs) and a powerful custom ${ }^{3} \mathrm{He} /{ }^{4} \mathrm{He}$ dilution refrigerator (DU).

\section{CUORE Initial Operations and Optimization}

The CUORE experiment started taking data in 2017. Detector optimization campaigns were performed in order to characterize and improve the detectors and overall system performance. This required a lot of effort since it was the first time that such a large number of macro-calorimeters were simultaneously operated in a completely new and unique cryogenic system.

\subsection{Noise Reduction}

The spectral shape of the noise in the CUORE detectors is the sum of several contributions, mainly vibration transmission from the cooling system: pulse tube-induced vibrations (peaks at $1.4 \mathrm{~Hz}$ and its harmonics), residual mechanical vibrations and oscillations related to the detector suspension and support structure $[11,12]$

A system to control the relative phases of the pressure oscillations of the PT cryocoolers was developed. Indeed pressure oscillations induce mechanical vibrations which are transmitted to the cryostat and eventually to the detectors. By driving the relative phases of the PTs, it is possible to find the configuration that maximizes the noise cancelation profiting of the interference between the PTs.

\subsection{Setting the Detector Operating Conditions}

The choice of the correct bias voltage is crucial for the operation of the CUORE NTD sensors. Therefore, special runs were acquired, in which the variation of the NTD resistance, signal amplitude and baseline noise were analyzed as a function of the applied voltage. The characteristic I-V curve (called "load curve") of each detector was measured and the best bias voltage, the so-called working point (WP), was then identified along the load curve. The optimal WP is usually a compromise between a large signal amplitude and the lowest noise condition, while still ensuring a linear and uniform behavior of the detector for small temperature variations. The load curve measurements and the identification of the optimal working points are, in 
general, procedures that can be performed manually in cryogenic experiments with a limited number of channels, like it was for CUORE-0. In CUORE, the number of channels to be optimized is almost one thousand; moreover, the NTD thermistors have resistances values higher and with a wider spread than small-size experiments. Therefore, it was necessary to develop new procedures and algorithms to automatize the load curve measurement and the working point identification for each operating temperature [13].

The system temperature has to be carefully regulated in order to successfully operate the CUORE detector array. Indeed it is crucial to identify the best operating temperature in order to achieve the highest quality readout with a good signal-tonoise ratio. Moreover, it is necessary to maintain this temperature stable over the course of a prolonged run. Temperature scans were taken in 2017 to select the best operational temperature that, together with the noise reduction, contributes to reach the CUORE goal of $5 \mathrm{keV}$ nominal energy resolution.

The working temperature was initially set at $15 \mathrm{mK}$ for the CUORE initial data taking in 2017. Based on the results of the temperature scans, it appeared that lower temperatures lead to a better energy resolution in the detector response to pulser events and particle signals. For this reason for the data taking from 2018, it was decided to set an operational temperature of $11 \mathrm{mK}$. Since the NTD working resistances at $11 \mathrm{mK}$ were higher than the nominal values proposed for CUORE, special care was taken in setting NTD operating conditions which could ensure a stable and uniform response in a wide energy range.

\section{CUORE Physics Data Taking}

The CUORE first science runs were carried out by mid-2017 at $15 \mathrm{mK}$ temperature. After the initial CUORE physics data taking, dedicated runs devoted to further characterization and optimization of the detector were performed in late 2017. In early 2018 , the system underwent a period of maintenance, during which the gate valves for the internal detector calibration system were replaced and an external system for calibration was installed. The physics data taking restarted in spring 2018 at $11 \mathrm{mK}$ operating temperature. The data taking proceeded up to late 2018 when the experiment experienced another warm-up at $100 \mathrm{~K}$, followed by the investigation of the leak in the cryostat causing the issue. A dedicated maintenance of the cryogenic system was performed in early 2019, in order to ensure a stable and long-term operation of the system. The data taking restarted in March 2019 and it is ongoing. CUORE acquired a total raw exposure (before analysis cuts) of more than $400 \mathrm{~kg}\left(\mathrm{TeO}_{2}\right)$ year up to July 2019.

The CUORE experiment released physics results come from the unblinded data from 2017. The first performed analysis consisted in the search of $0 v \beta \beta$ decay of $\mathrm{TeO}_{2}$, and afterward, the analysis was focused on the evaluation of the $2 \nu \beta \beta$ half-life and background model reconstruction $[14,18]$. The total collected exposure (after the analysis selections) was $86.3 \mathrm{~kg}\left(\mathrm{TeO}_{2}\right)$ year for the 2017 data. 


\subsection{Search for $0 v \beta \beta$ of ${ }^{130} \mathrm{Te}$}

The CUORE physics energy spectra, in which to look for a possible $0 v \beta \beta$ signal of ${ }^{130} \mathrm{Te}$, were obtained after applying basic quality cuts, rejection of spurious signals by pulse shape analysis and anti-coincidence selections to the raw data. Science data were blinded until all the selections, and fit procedures had been fixed.

The average energy resolution at $Q_{\beta \beta}$ in the science runs was $(7.7 \pm 0.5) \mathrm{keV}$ FWHM, almost reaching the $5 \mathrm{keV}$ CUORE goal.

The background index in the ROI was obtained via fitting the region with no $0 v \beta \beta$ component in the model: $b=(0.014 \pm 0.002)$ counts/(keV $\mathrm{kg}$ year $)$, which was consistent with the expectations given the CUORE background budget.

The complete model for the ROI including of the $0 v \beta \beta$ component was utilized for performing a fit in the region around $Q_{\beta \beta}$ : There was no evidence for $0 v \beta \beta$ decay. Including the systematic uncertainties, the $90 \%$ lower limit on the half-life was extracted: $T_{1 / 2}^{0 v}\left({ }^{130} \mathrm{Te}\right)>1.3 \times 10^{25}$ year $(90 \%$ C.L. $)$.

The CUORE result was combined with the CUORE-0 [15, 16] and Cuoricino [17] results: $T_{1 / 2}^{0 v}\left({ }^{130} \mathrm{Te}\right)>1.5 \times 10^{25}$ year (at 90\% C.L.) [14]. A limit on the effective Majorana neutrino mass $\left(m_{\beta \beta}\right)$ in $0 v \beta \beta$ decay models mediated by a light Majorana neutrino exchange can be extracted from the half-life limits: $m_{\beta \beta}<110-520 \mathrm{meV}$ (at $90 \%$ C.L.). In Fig. 2, the current experimental limits on $m_{\beta \beta}$ are shown. The regions of $m_{\beta \beta}$ allowed by oscillations are shown for both inverted and normal hierarchies of neutrino mass. The horizontal bands with arrows indicate the most stringent upper

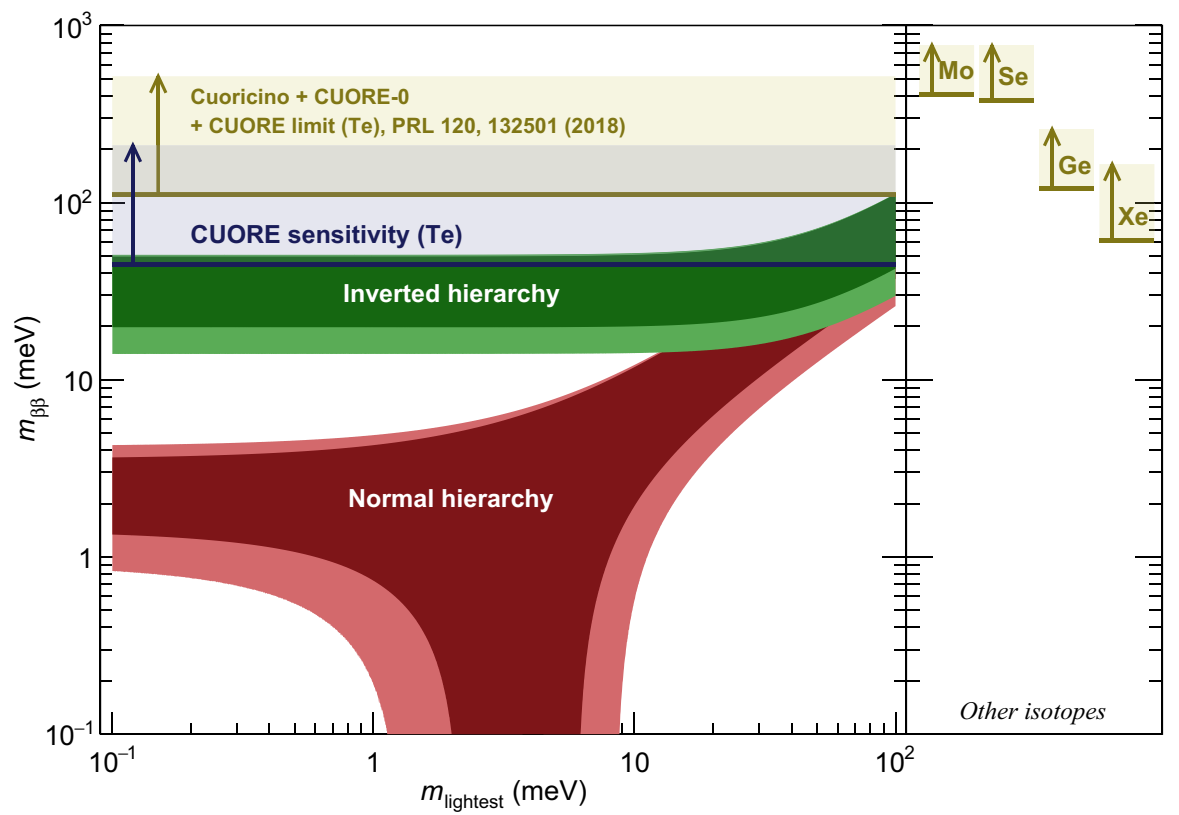

Fig. 2 Experimental limits on $m_{\beta \beta}$ and current CUORE result and sensitivity [14] (Color figure online) 
limits on $m_{\beta \beta}$ coming from the experimental searches of $0 v \beta \beta$ with several isotopes, including the results for ${ }^{130} \mathrm{Te}$ from CUORE combined with CUORE-0 and Cuoricino; moreover, the CUORE sensitivity on $m_{\beta \beta}$ for 5 years of data taking is shown.

\subsection{Measurement of $2 v \beta \beta$ half-life of ${ }^{130} \mathrm{Te}$}

Given the physics data acquired in 2017 , the analysis to reconstruct the observed CUORE background and extract the ${ }^{130} \mathrm{Te} 2 v \beta \beta$ half-life was also performed [18].

The physics data were split into four types of spectra: by-multiplicity and bylayer. Single-detector events fill the multiplicity 1 (M1) spectrum, while double-hit events are assigned with multiplicity 2 (M2). The M1 spectrum is also split in two geometrical layers: the 12 outer towers are more sensitive to contaminations on the shields (outer layer, L1) and the 9 inner towers to contaminations on the crystals (inner layer, L0).

A set of 60 sources, considering different isotopes and locations, was sufficient to reconstruct the CUORE observed physics data, via a Markov Chain Monte Carlo simultaneous fit across the four energy spectra [19]. Many contaminations were identified and located, and others were still utilized by the fit, but only a limit on their activity was obtained. The model was able to reproduce nearly all the major features of the observed spectra. The $2 v \beta \beta$ decay is the dominant component of the observed spectrum for single-site events between 1 and $2 \mathrm{MeV}$ (see Fig. 3). The $2 v \beta \beta$ half-life of ${ }^{130} \mathrm{Te}$ was obtained: $T_{1 / 2}^{2 v}\left({ }^{130} \mathrm{Te}\right)=[7.9 \pm 0.1$ (stat) \pm 0.2 (syst) $] \times 10^{20}$ year. The result is consistent with previous measurements of the same half-life [20, 21]. It represents the most precise measurement of ${ }^{130} \mathrm{Te} 2 v \beta \beta$ half-life.

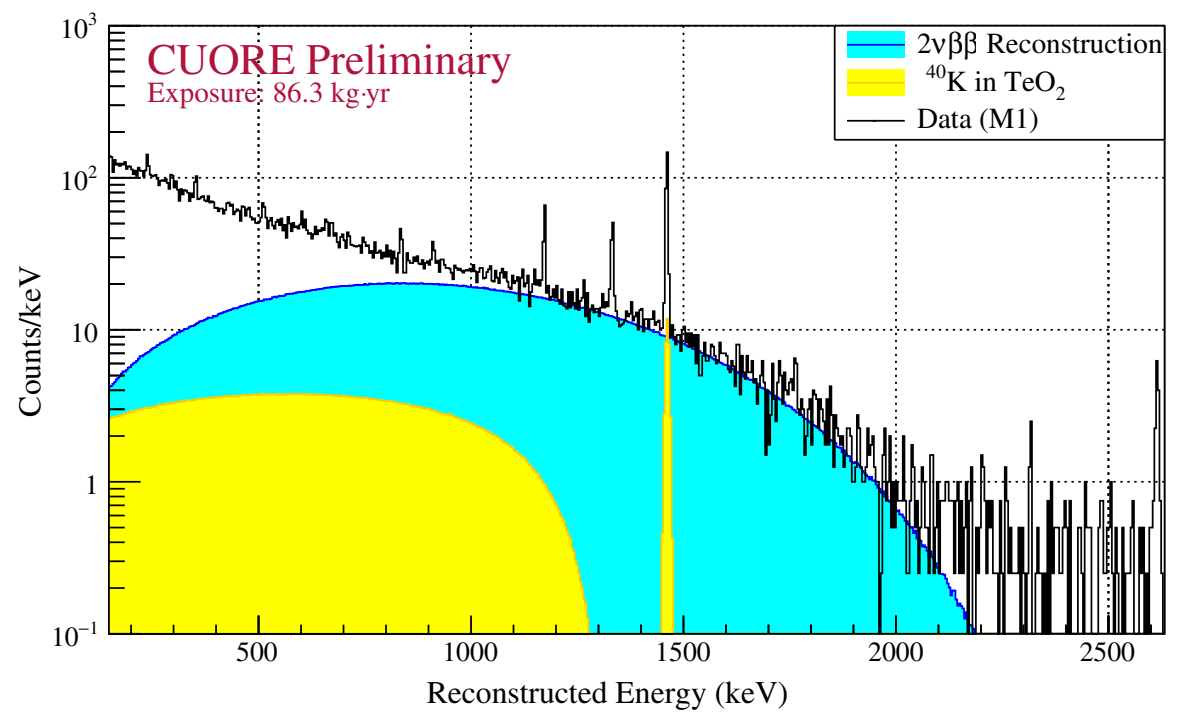

Fig. 3 CUORE observed spectrum (black) of multiplicity 1 events in the inner layer (M1L0), with a reconstruction of the $2 v \beta \beta$ component (cyan) of the background as well as the ${ }^{40} \mathrm{~K}$ background (yellow) [18] (Color figure online) 


\section{Conclusions}

CUORE is the first ton-scale operating cryogenic calorimetric $0 v \beta \beta$ detector. The first CUORE physics results of $0 \nu \beta \beta$ and $2 \nu \beta \beta$ in ${ }^{130} \mathrm{Te}$ were obtained from the analysis of physics data collected in 2017 for $86.3 \mathrm{~kg}\left(\mathrm{TeO}_{2}\right)$ year exposure. A total raw exposure of more than $400 \mathrm{~kg}$ year was already available in July 2019; updated results for $372.5 \mathrm{~kg}\left(\mathrm{TeO}_{2}\right)$ year exposure (after the analysis selections) were released in December 2019 [22].

The CUORE data taking is currently underway to collect 5 years of run-time. While proceeding with the physics data taking, CUORE keeps studying and testing new strategies to improve the detector energy resolution and to better understand the sources of background and their effect of the measured energy spectra.

Acknowledgements The author thanks the CUORE collaboration, the directors and staff of the Laboratori Nazionali del Gran Sasso and the technical staff of the laboratories. This work was supported by the Istituto Nazionale di Fisica Nucleare (INFN); the European Research Council; the National Science Foundation (NSF); the US Department of Energy (DOE) Office of Science; and the DOE Office of Nuclear Physics.

\section{References}

1. M. Tanabashi et al., Particle data group. Phys. Rev. D 98, 030001 (2018)

2. M.J. Dolinski et al., Annu. Rev. Nucl. Part. Sci. 69, 219 (2019)

3. S. Dell'Oro et al., Adv. High Energy Phys. 2016, 2162659 (2016)

4. D.R. Artusa et al., (CUORE collaboration), Adv. High Energy Phys. 2015, 879871 (2015)

5. C. Alduino et al., (CUORE collaboration), Eur. Phys. J. C 77, 532 (2017)

6. E. Fiorini, T. Niinikoski, Nucl. Instrum. Meth. 224, 83 (1984)

7. C. Alduino et al., (CUORE collaboration), J. Inst. 11(07), P07009 (2016)

8. P. Carniti et al., J. Instrum. 13, P02029 (2018)

9. A. D'Addabbo et al., J. Low Temp. Phys 193, 867-875 (2018)

10. C. Alduino et al., Cryogenics 102, 9 (2019)

11. A. D'Addabbo et al., Cryogenics 93, 55-56 (2018)

12. A. Bersani et al., The detector suspension system of the CUORE experiment (in preparation)

13. I. Nutini et al., An highly automated system to set working points for large bolometric arrays (in preparation)

14. C. Alduino et al., (CUORE collaboration), Phys. Rev. Lett. 120, 132501 (2018)

15. K. Alfonso et al., (CUORE collaboration), Phys. Rev. Lett. 115, 102502 (2015)

16. C. Alduino et al., (CUORE collaboration), Phys. Rev. C 93, 045503 (2016)

17. E. Andreotti et al., Astropart. Phys. 34, 822 (2011)

18. D.Q. Adams et al. (CUORE collaboration) Update on the recent progress of the CUORE experiment. arXiv:1808.10342 (2018)

19. C. Alduino et al. (CUORE collaboration), Eur. Phys. J. C77, 8, 543 (2017)

20. C. Alduino et al. (CUORE collaboration), Eur. Phys. J. C77, 1, 13, (2017)

21. R. Arnold et al., NEMO-3 collaboration. Phys. Rev. Lett. 107, 062504 (2011)

22. D.Q. Adams et al. (CUORE collaboration), Improved limit on neutrinoless double-beta decay in 130Te with CUORE. arXiv:1912.10966 (2019)

Publisher's Note Springer Nature remains neutral with regard to jurisdictional claims in published maps and institutional affiliations. 


\section{Affiliations}

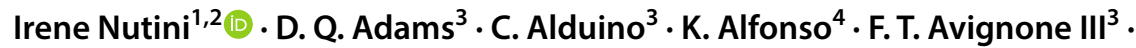

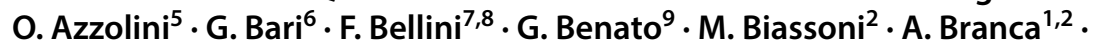
C. Brofferio ${ }^{1,2}$. C. Bucci ${ }^{10}$ - A. Caminata ${ }^{11}$ - A. Campani ${ }^{11,12}$ - L. Canonica ${ }^{10,13}$.

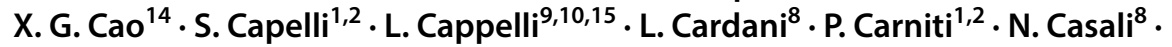
D. Chiesa ${ }^{1,2} \cdot \mathrm{N}$ Chott $^{3} \cdot$ M. Clemenza ${ }^{1,2} \cdot$ S. Copello ${ }^{10,16} \cdot$ C. Cosmelli ${ }^{7,8}$. O. Cremonesi ${ }^{2}$. R. J. Creswick ${ }^{3}$. A. D'Addabbo ${ }^{10}$. D. D'Aguanno ${ }^{10,17}$. I. Dafinei ${ }^{8}$. C. J. Davis ${ }^{18} \cdot$ S. Dell'Oro ${ }^{19} \cdot$ S. Di Domizioo ${ }^{11,12} \cdot$ V. Dompè ${ }^{10,16}$. D. Q. Fang ${ }^{14}$. G. Fantini ${ }^{10,16} \cdot$ M. Faverzani ${ }^{1,2} \cdot$ E. Ferri $^{1,2} \cdot$ F. Ferroni $^{8,16} \cdot$ E. Fiorini $^{1,2}$. M. A. Franceschi ${ }^{20}$ - S. J. Freedman ${ }^{9,15}$ - B. K. Fujikawa ${ }^{15}$ - A. Giachero ${ }^{1,2}$. L. Gironi ${ }^{1,2} \cdot$ A. Giuliani ${ }^{21} \cdot$ P. Gorla ${ }^{10} \cdot$ C. Gotti ${ }^{1,2}$-T. D. Gutierrez ${ }^{22} \cdot$ K. Han $^{23}$. K. M. Heeger ${ }^{18} \cdot$ R. G. Huang ${ }^{9} \cdot$ H. Z. Huang ${ }^{4} \cdot$ J. Johnston ${ }^{13} \cdot$ G. Keppel ${ }^{5}$.

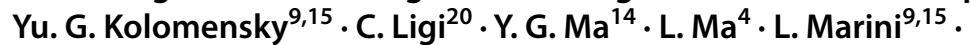
R. H. Maruyama ${ }^{18} \cdot$ Y. Mei $^{15} \cdot \mathrm{N}$. Moggi $^{6,24} \cdot$ S. Morganti ${ }^{8} \cdot$ T. Napolitano $^{20}$. M. Nastasi ${ }^{1,2}$. J. Nikkel ${ }^{18}$. C. Nones ${ }^{25}$. E. B. Norman ${ }^{26,27}$. V. Novati ${ }^{21}$. A. Nucciotti ${ }^{1,2} \cdot$ T. O'Donnell ${ }^{19} \cdot$ J. L. Ouellet ${ }^{13} \cdot$ C. E. Pagliarone ${ }^{10,17}$.

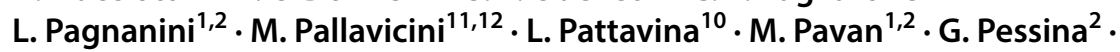
V. Pettinacci ${ }^{8} \cdot$ C. Pira ${ }^{5}$. S. Pirro ${ }^{10}$ - S. Pozzi ${ }^{1,2} \cdot$ E. Previtali $^{2} \cdot$ A. Puiu ${ }^{1,2}$. C. Rosenfeld ${ }^{3}$. C. Rusconi ${ }^{3,10} \cdot$ M. Sakai $^{9} \cdot$ S. Sangiorgio ${ }^{26} \cdot$ B. Schmidt ${ }^{15}$. N. D. Scielzo ${ }^{26} \cdot$ V. Singh ${ }^{9} \cdot$ M. Sisti ${ }^{1,2} \cdot$ D. Speller ${ }^{18} \cdot$ L. Taffarello $^{28}$.

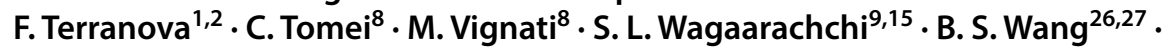
B. Welliver ${ }^{15} \cdot$ J. Wilson $^{3} \cdot \mathrm{K}_{\text {. Wilson }}{ }^{3} \cdot$ L. A. Winslow ${ }^{13} \cdot$ L. Zanotti $^{1,2}$. S. Zimmermann ${ }^{29} \cdot$ S. Zucchelli ${ }^{6,24}$

\footnotetext{
1 Dipartimento di Fisica, Università di Milano-Bicocca, 20126 Milan, Italy

2 INFN - Sezione di Milano Bicocca, 20126 Milan, Italy

3 Department of Physics and Astronomy, University of South Carolina, Columbia, SC 29208, USA

4 Department of Physics and Astronomy, University of California, Los Angeles, CA 90095, USA

5 INFN - Laboratori Nazionali di Legnaro, 35020 Legnaro, Padua, Italy

6 INFN - Sezione di Bologna, 40127 Bologna, Italy

7 Dipartimento di Fisica, Sapienza Università di Roma, 00185 Rome, Italy

$8 \quad$ INFN - Sezione di Roma, 00185 Rome, Italy

9 Department of Physics, University of California, Berkeley, CA 94720, USA

10 INFN - Laboratori Nazionali del Gran Sasso, 67100 Assergi, L’Aquila, Italy

11 INFN - Sezione di Genova, 16146 Genoa, Italy

12 Dipartimento di Fisica, Università di Genova, 16146 Genoa, Italy

13 Massachusetts Institute of Technology, Cambridge, MA 02139, USA

14 Shanghai Institute of Applied Physics, Chinese Academy of Sciences, Shanghai 201800, China

15 Nuclear Science Division, Lawrence Berkeley National Laboratory, Berkeley, CA 94720, USA

16 INFN - Gran Sasso Science Institute, 67100 L'Aquila, Italy
} 
17 Dipartimento di Ingegneria Civile e Meccanica, Università degli Studi di Cassino e del Lazio Meridionale, 03043 Cassino, Italy

18 Wright Laboratory, Department of Physics, Yale University, New Haven, CT 06520, USA

19 Center for Neutrino Physics, Virginia Polytechnic Institute and State University, Blacksburg, VA 24061, USA

20 INFN - Laboratori Nazionali di Frascati, 00044 Frascati, Rome, Italy

21 CSNSM, Univ. Paris-Sud, CNRS/IN2P3, Université Paris-Saclay, 91405 Orsay, France

22 Physics Department, California Polytechnic State University, San Luis Obispo, CA 93407, USA

23 INPAC and School of Physics and Astronomy, Shanghai Jiao Tong University; Shanghai Laboratory for Particle Physics and Cosmology, Shanghai 200240, China

24 Dipartimento di Fisica e Astronomia, Alma Mater Studiorum - Università di Bologna, 40127 Bologna, Italy

25 Service de Physique des Particules, CEA/Saclay, 91191 Gif-sur-Yvette, France

26 Lawrence Livermore National Laboratory, Livermore, CA 94550, USA

27 Department of Nuclear Engineering, University of California, Berkeley, CA 94720, USA

28 INFN - Sezione di Padova, 35131 Padua, Italy

29 Engineering Division, Lawrence Berkeley National Laboratory, Berkeley, CA 94720, USA 